\title{
Jubilee Editorial
}

\section{Auscultation and phonocardiography: a personal view of the past 40 years}

\author{
AUBREY LEATHAM \\ From the London, National Heart, and St George's Hospitals, London
}

The establishment of the British Cardiac Society coincided with and fostered the golden era of clinical cardiology that had staried in the United Kingdom with Sir James Mackenzie and his school and reached its peak with Paul Wood in the 1950s. Factors known to me which influenced the spectacular advances in clinical diagnosis were the accuracy of postmortem examinations at the Bernhard Baron Institute of Pathology at the London Hospital under the direction of Professor Turnball (this was continued by Reginald Hudson at the National Heart Hospital and later by Michael Davies at St George's Hospital); accurate diagnosis by cardiac radiology led by John Parkinson; and the haemodynamic findings of right heart catheterisation which had been introduced by Cournand in the United States and by Sharpey-Schafer, McMichael, and Wood in England.

The first specialised cardiac department in a general hospital in England, and perhaps in the world, was started by Mackenzie at the London Hospital in 1911. He was followed by John Parkinson (later knighted for his service to medicine) in 1920, who all his life successfully fought to advance cardiology as a specialty. In 1931, with the help of the local traders and a gift of $£ 500$ from Queen Mary, a new cardiac department was opened at the London Hospital. In it Parkinson installed an $x$ ray screening unit and pioneered the development of cardiac radiology. In 1947 one of his patients, William Sherbrook, left sufficient funds to start a new research fellowship and I was the first recipient.

In 1946 I was resident medical officer at the National Heart Hospital. John Parkinson and William Evans had been the mainstay of the hospital during the second world war and when I arrived at the end of the war I found a new atmosphere of questioning research which I had not encountered before. For example, I was perplexed by the vari-

Requests for reprints to Dr Aubrey Leatham, 45 Wimpole Street, London W1M 7DG. ations in the clinical significance of hypertension and wondered whether examination of the fundi would help.I was immediately encouraged to start a survey of the clinical value of the retinal vessels in the assessment of hypertension and I spent many hours in the dark in Parkinson's screening room examining the fundi of his patients whom I was not allowed to examine in daylight lest the findings should bias my observations.

Auscultation and phonocardiography started for me with the gardener at the country branch of the hospital who while tending his plants was noticeably short of breath. When I examined him I found very abnormal heart sounds which I could not interpret. An earphone harnessed to the hospital string galvanometer electrocardiograph produced puzzling and large low frequency vibrations not at all resembling auscultation. A chest radiograph showed that he had an enormous heart; he died six months later and at necropsy proved to have Lutembacher's syndrome.

In 1947 as a specialist physician in the Army my main commitment was as mess secretary and I was therefore overjoyed to receive an offer from Parkinson and Evans to be the first Sherbrook Research Fellow at the London Hospital-I was informed that early release from the Army was available for research work (and coal mining). William Evans (fig 1) had made a start on recording heart sounds and murmurs using an old Cambridge double string galvanometer. His recordings were dominated by huge low frequency oscillations bearing no relation to the auscultatory findings (fig 2).

Rappaport and Sprague had shown in 1941 that considerable filtration of the low frequencies was required for the results of the phonocardiogram to resemble auscultation. This seemed to be the first step if the graphic recordings were to clarify and improve auscultation, which at that time was largely discarded as a scientific diagnostic method, an important reason being that Sir Thomas Lewis had little time for it. He had stated that the murmur of 

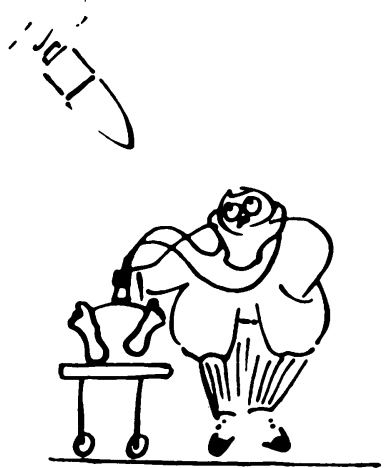

\section{Do I hear more than two sounds?}

Fig 1 William Evans at work. A caricature which appeared in The London Hospital Gazette during the heavy bombing of London in 1941.

mitral stenosis must be recognised by memorising its characteristics like the bark of a dog, and he and all the other senior physicians completely disregarded systolic murmurs. I was impressed by the American physiologist Wiggers who had demonstrated asynchrony between left and right ventricular pressure pulses and by Wolferth and Margolies who in 1935 had identified the two components of the second heart sound in bundle branch block by using a simultaneous carotid pulse, and had thereby reversed the electrocardiographic interpretation of the bundle affected. Dock had popularised the hypothesis that the opening and closing of valves was the major cause of heart sounds. It seemed to me that filtration of the low frequencies as described by Rappaport and Sprague would not only have the clinical advantage of producing results resembling auscultation, but should also show high frequency transients synchronising with valve movement (if this were the cause of the sounds) and would make identification of the components of sounds relatively easy. Fortunately, I was not aware that I would have

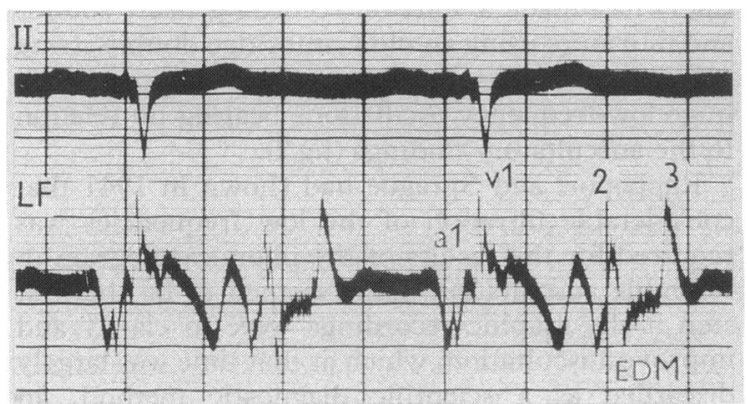

Fig 2 Phonocardiography without filters as practised by William Evans in the early 1940s. to wait for thirty years for echocardiography to prove this correct.

Doctor Bernstein in the Physiology Department of the London Hospital made for us amplifiers with suitable filters, and Malcolm Towers and I started work on the second heart sound, because we could hear inspiratory splitting, just as described by Potain in 1866, and this seemed to be the first step in establishing that there was asynchronous contraction and relaxation of the two sides of the heart. I later realised that a study of the second heart sound was a particularly appropriate first step because James Hope, cardiologist to St George's Hospital, had been the first to show that the second heart sound was related to closure of the aortic and pulmonary valves. Using the double string galvanometer we made simultaneous phonocardiograms from the pulmonary area and mitral area together with an electrocardiogram or a recording of the carotid pulse. The first component of the second sound was the major one; it timed with the dicrotic notch of the carotid pulse, and was the sole component at the apex. The second component was confined to the pulmonary area and nearby (fig 3), and its delay during inspiration was the major cause of physiological splitting of the second sound. The weakness of our recording system was the tendency

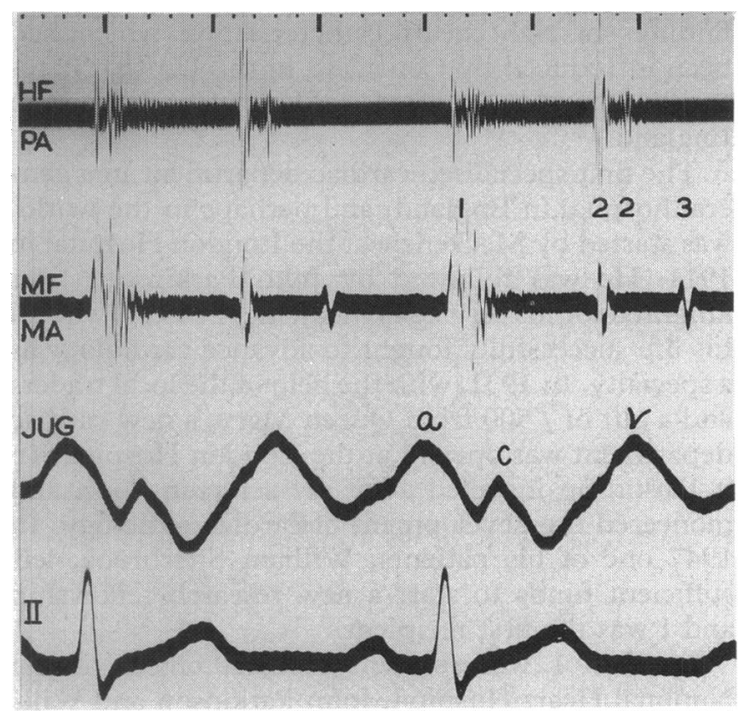

Fig 3 Simultaneous phonocardiograms from the pulmonary area $(P A)$ and mitral area $(M A)$ showing that the later component of the second sound was confined to the pulmonary area. Filters were used to cut out the low frequencies and transmit the high frequencies so that the vibrations recorded resembled the results of auscultation through a rigid diaphragm chest piece ( high frequency phonocardiogram $(H F)$ ), or through a bell (medium frequency phonocardiogram (MF)). 
for one string of our double string galvanometer to wrap itself around the other whenever we made a big deflection; this meant numerous trips to Cambridge in a 1934 baby Austin to visit an old technician who alone was able to unwrap the strings.

Elsewhere in Europe, and particularly in Sweden and Germany, the Mannheimer system with one microphone and multiple simultaneous channels each with a different frequency range was being developed, but nothing important was discovered with this technique (fig 4). Luisada in Chicago was using low frequency phonocardiograms (fig 5), bearing no relation to auscultation, and multiple timing channels with variable delays.

At the London Hospital cardiac radiology under Parkinson was making big advances. Each outpatient had to wait until the end of the afternoon for the $x$ ray screening session and Parkinson's time in the dark room would probably last for about an hour with discerning and entertaining comment on almost every patient. With the help of the radiological findings described by Parkinson, Papp, and Bedford in 1939 in an early issue of the British Heart Journal it was now possible to diagnose atrial septal defect. Barber, Magidson, and Wood had described the signs and we were looking at the peculiar second

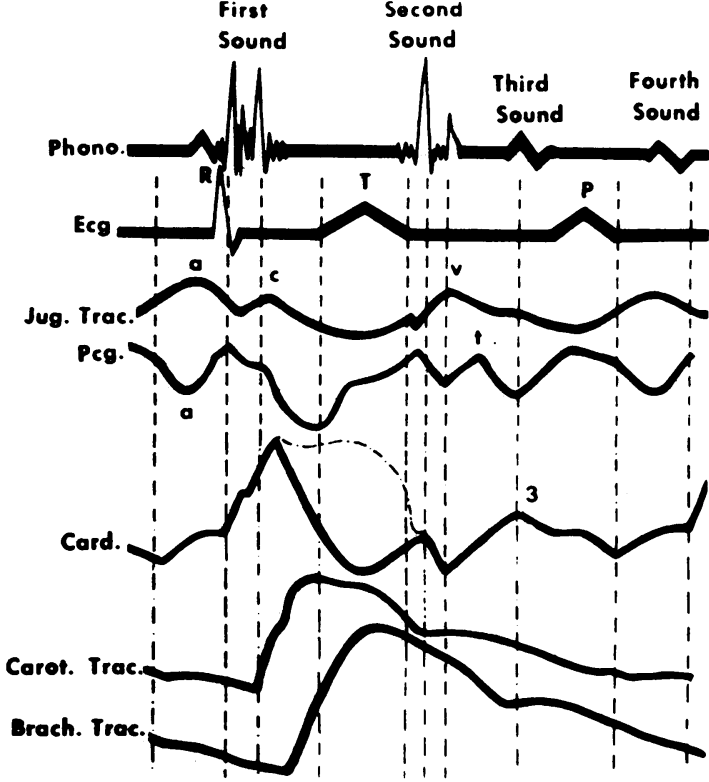

Fig 5 One of Luisada's fine drawings of a single low frequency phonocardiogram with no accurate timing channel.

\section{ECG}

earlike

\section{$25 \mathrm{~Hz}$}

$50 \mathrm{~Hz}$

$100 \mathrm{~Hz}$
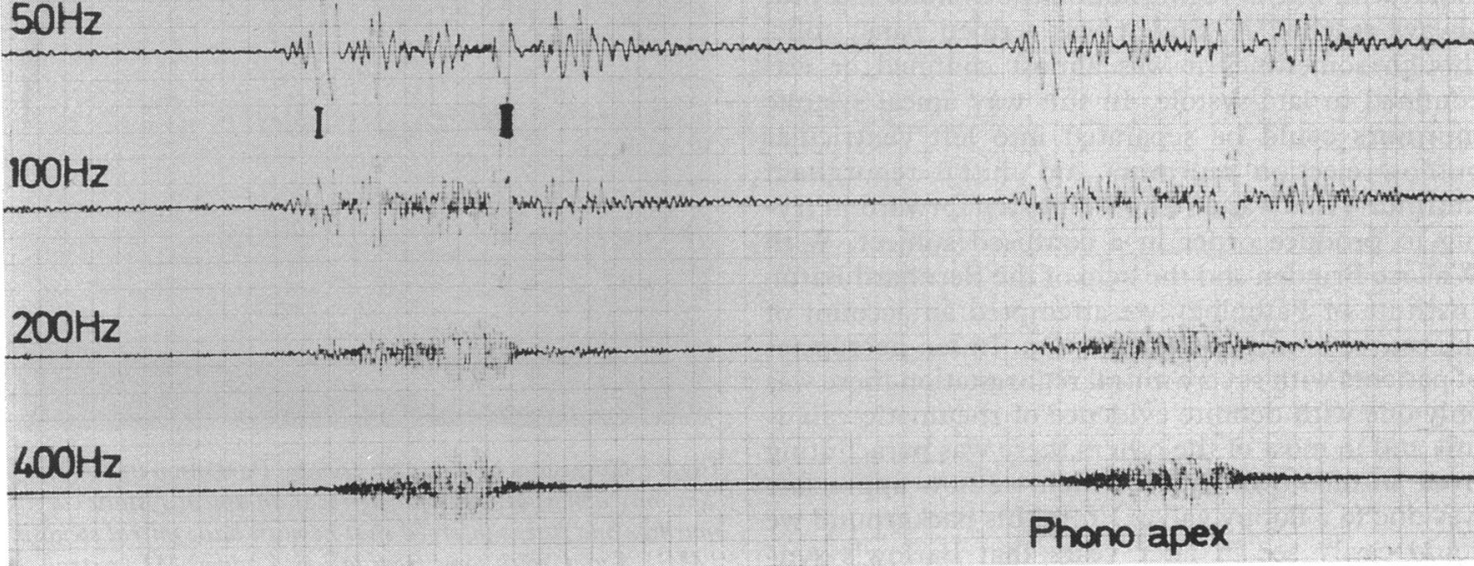

Fig 4 Multiple recording with varying filtration taken from a single microphone as developed by Mannheimer. 
heart sound. It was Bill Dicks, our technician and collaborator at the London Hospital who said triumphantly one day, looking at the moving shadow of the string rather than at the finished graph, that the "split was fixed". Measurements proved him right, showing that both $A_{2}$ and $P_{2}$ were delayed nearly equally on inspiration. The description of reversed splitting of the second sound in left bundle branch block was an easy step from knowledge of physiological splitting. The discoveries that the delay of $\mathrm{P}_{2}$ gave an exact estimate of severity of pulmonary stenosis and that the Eisenmenger ventricular septal defect and single ventricle gave a single sound had to wait for the stimulation of Paul Wood at the National Heart Hospital.

At the London Hospital the influence of Mackenzie, and now Parkinson, had resulted in systolic murmurs being almost ignored and certainly being regarded as unimportant. This was an overreaction to the diagnostic abuse and unnecessary cardiac invalidism (one of William Evans's famous phrases) that in earlier years had been prompted by the presence of a systolic murmur. Parkinson's reaction to a systolic murmur is demonstrated by the case notes (fig 6) of a patient with a systolic murmur (fig 7). (Indeed William Evans said of mitral incompetence that it was a description of the physician who diagnosed it; one wonders what he would say of mitral valve prolapse.) Thirty years later the patient with the systolic murmur died of coronary disease and proved to have mild rheumatic mitral regurgitation. It was soon clear from our high frequency phonocardiograms that the systolic murmur of aortic stenosis finished before the aortic component of the second heart sound, as indeed it had to, bearing in mind the cessation of forward flow before closure of the valve, and it seemed logical to describe these and similar murmurs as ejection murmurs. On the other hand, with mitral regurgitation the systolic murmur always reached $\mathrm{A}_{2}$ and it was termed pansystolic, though sometimes it was almost confined or was confined to late systole. In this way apical systolic murmurs could be separated into left ventricular outflow ejection murmurs and mitral regurgitant murmurs; this was an important step forward in trying to produce order in a confused subject. With Wallace Brigden and the help of the Bernhard Baron Institute of Pathology we attempted an account of the course of mitral regurgitation. In ten necropsies of patients with severe mitral regurgitation there was only one with definite evidence of rheumatic valvulitis and in most of the others there was parachuting from an unknown cause, which we now appreciate was due to a floppy valve. From this background we could easily see in later years that Barlow's syndrome and mitral valve prolapse and the threat of sudden death would prove to be a fiasco in all but the most exceptional cases.

I moved in 1951 to the National Heart Hospital where Paul Wood was Director of the Institute of Cardiology. The chief technician John Norman found for me another 1918 Cambridge table model string electrocardiograph on which we mounted a second string and two mirror galvanometers with the aid of the Cambridge Instrument Company (fig 8). Each of the four galvanometers had a separate light source and these four sources had to be matched up to obtain an evenly illuminated recording; this was particularly difficult because the colour of the light sources varied. The falling plate camera used film, which was far superior to the usual recording paper because of its bigger density range. The length of the recording was limited to $15 \mathrm{~cm}$, but the speed of fall of the plate could be varied infinitely to suit the requirements of the particular recording, and this system was ideal for composing a suitable length of recording to fit a page of the Brit-

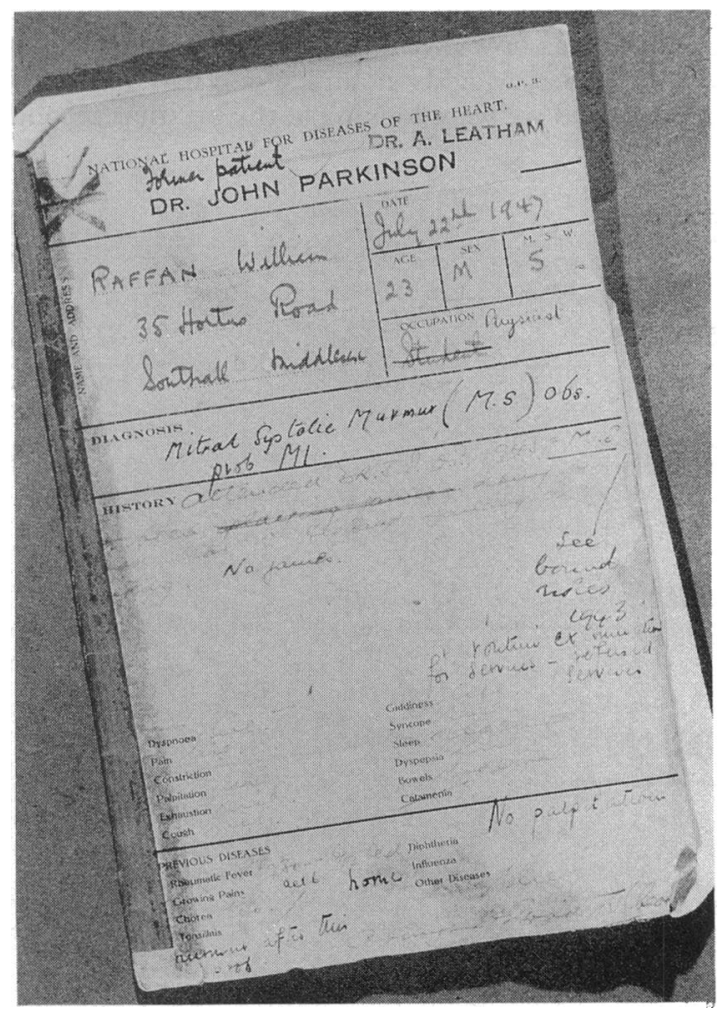

Fig 6 Case notes of a patient seen by Parkinson in 1947. The only abnormal physical sign was an apical systolic murmur and it was inferred that he must have mitral stenosis (MS). Later, with some trepidation, I added MI (mitral incompetence). 


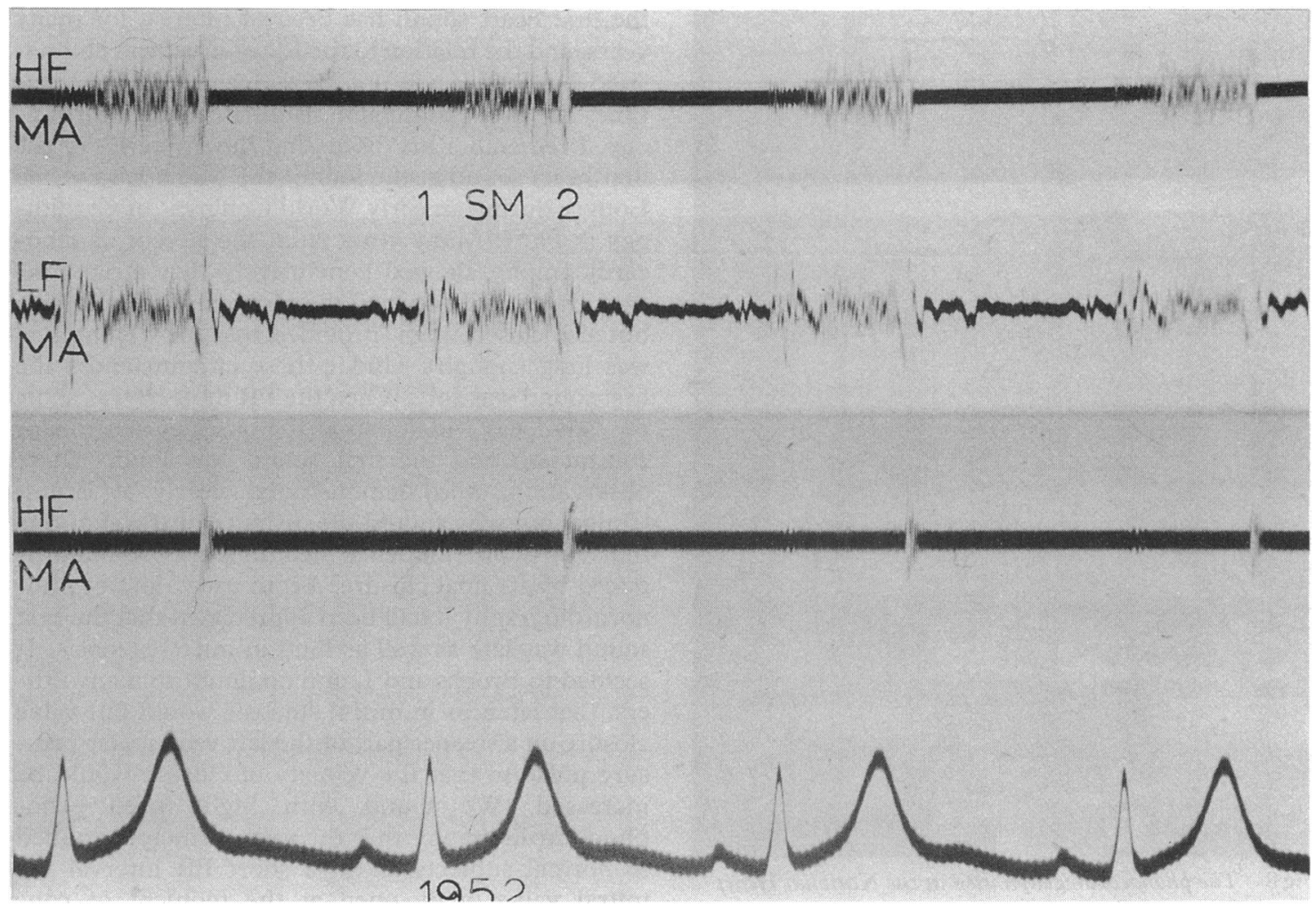

Fig 7 Phonocardiogram of same patient in 1952 showing a pansystolic murmur and third sound.

ish Heart Journal. The quality of the sound recordings made with this apparatus has never been surpassed (fig 9); it cannot even be equalled with modern apparatus.

The final and most difficult problem was identification of the components of the first heart sound and their separation from the early systolic ejection sounds. Right from the beginning of our recordings of the high frequency components of the first sound at the London Hospital it was clear that there were two components and, bearing in mind the precedence of left ventricular contraction over right, it seemed reasonable to guess that the first component arose from mitral valve closure and the second from tricuspid. Furthermore, the first component was by far the larger and was maximal at the apex, as befitting mitral closure, while the second was too late for mitral closure in relation to the carotid upstroke and was maximal at the lower left sternal edge over the tricuspid valve. Working with Edgar Haber, who was nearly as good at auscultation as at immunoassay, we had support for this concept by finding further delay in the second component with right bundle branch block, left ventricular ectopic complexes, and pacing with the electrode attached to the left ventricle. Opposition came from Luisada and his group in Chicago who could not record tricuspid closure with intracardiac phonocardiograms; also destruction of the right ventricle in dogs failed to alter the first sound which they thought came entirely from the left side of the heart. When they found that the pressure crossovers of the ventricles and atria occurred before the sound, they deduced that valve closure was noiseless. They were seemingly unaware of the lag between a pressure crossover and final halt of the valve. Even the Pittsburg group led by Shaver with superb sound pressure correlates denied a tricuspid origin to the second component of the first sound and attributed this sound to aortic ejection ("root sound"), even though the ejection sound in aortic stenosis was later and related to aortic valve opening.

We had to await echocardiography to obtain the exact timing of valve movement, but in the early days it was difficult to obtain reflection from the normal tricuspid valve and so we chose patients with Ebstein's anomaly and a large anterior leaflet of the tricuspid valve. The loud late component of the first sound, which we had assumed to be tricuspid closure delayed by the almost invariable right bundle 


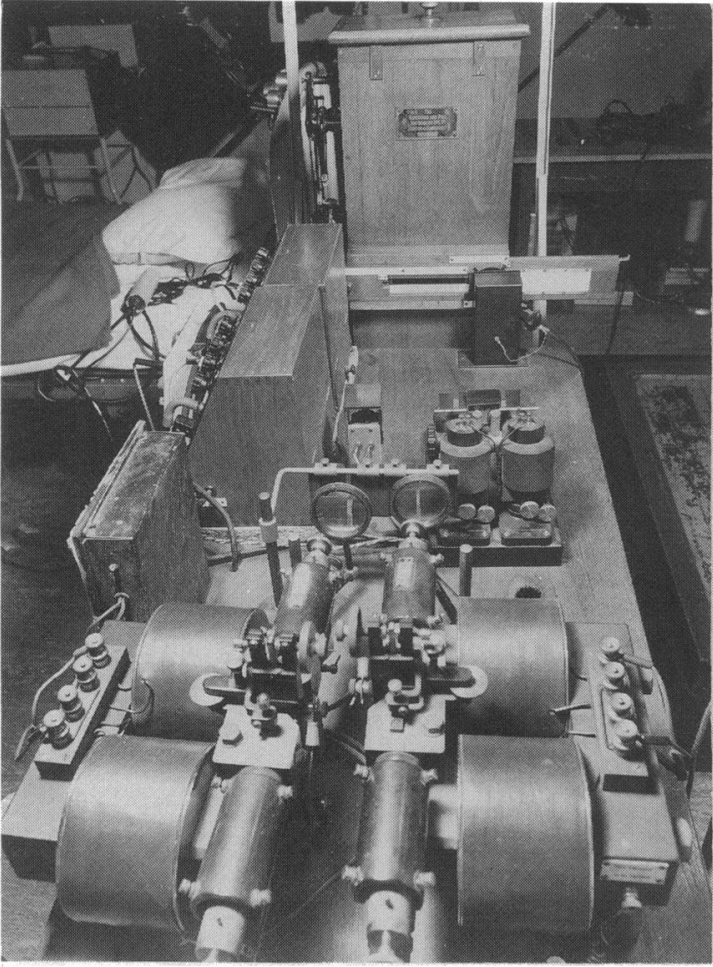

Fig 8 The phonocardiograph used at the National Heart Hospital from 1952 onwards.

branch block, was shown to coincide exactly with the final halt of the tricuspid valve on Dr Pridie's pioneering apparatus. Subsequently, with apparatus improved by Graham Leech, and simultaneously with Ernest Craige of Chapel Hill (and St George's Hospital), it was demonstrated that splitting of the first sound, as heard in most normal subjects by means of a rigid-diaphragm chest piece at the lower left sternal edge, was indeed due to mitral closure followed by tricuspid closure. There was synchrony between the final halt of the closing movement of the leaflets on the echocardiogram and the first component of the first heart sound and that of the closing tricuspid valve and the second component. This led to work with Nicholas Brooks and Mark Dancy on bundle branch block; it was confirmed that tricuspid closure was late in patients with isolated right bundle branch block; however, in patients with right bundle branch block and symptoms from generalised conducting tissue disease closure of the tricuspid valve was not delayed, indicating that the block was peripheral and occurred at arborisation level. This accorded with the site of histological change that was demonstrated by Michael Davies.

The mechanism causing variation in intensity of the first heart sound has been of interest for many years, and the relation to the PR interval was emphasised by Sam Levine of Boston, who during these times had done so much to stimulate clinical cardiology. I remember his discussing the mysteries of the first heart sound at the foot of the Mona Lisa in the Louvre during an early World Congress of Cardiology in Paris. Many years later, the advent of echocardiography showed conclusively that atrial contraction not only opened the atrioventricular valves, but also closed them (provided that the PR interval was long enough). Under these circumstances the first sound was soft. When the PR interval was short the valve was wide open at the onset of ventricular contraction and the first sound was loud. These observations were demonstrated clearly by Ernest Craige, but why should the distance of travel of this flimsy structure influence the intensity of sound produced by its final closure? From early days of phonocardiography it had been appreciated that the first sound was late as well as loud in mitral stenosis. It seemed to Brooks and I, and no doubt to many others, that lateness in mitral stenosis would put valve closure on a steeper part of the left ventricular pressure pulse so that the velocity of closure would be increased. We found with high speed echophonocardiography that the same principle applied to normal subjects. With a short PR interval the mitral valve, wide open at the moment of contraction, was late in reaching its final halting point and this coincided with a loud first sound. With a long PR interval the valve was nearly closed at the moment of ventricular contraction and its final movement and halt occurred at the beginning of ventricular contraction and coincided with a soft first sound.

Finally, a few words about early systolic sounds which Vogelpoel and I termed ejection sounds, despite the superb French expression "claguement protosystolique". Although the early ejection sound in pulmonary valve stenosis and the later ejection sound in pulmonary root dilatation from pulmonary hypertension with its prolonged ventricular isovolumic time had been useful in diagnosis, there was great debate about aortic ejection sounds. In the early days before the advent of echocardiography we were puzzled by the lateness of the aortic ejection sound which occurred during the upstroke of the aortic pressure pulse and not earlier when the valve opened. We had already used cineangiocardiography to examine the mitral valve and had showed that its final halt coincided with the loud first heart sound of mitral stenosis and we were contemplating a similar technique for the aortic valve when Richard Ross and O'Neil Humphries (exfellow from St George's) gave us the answer from 


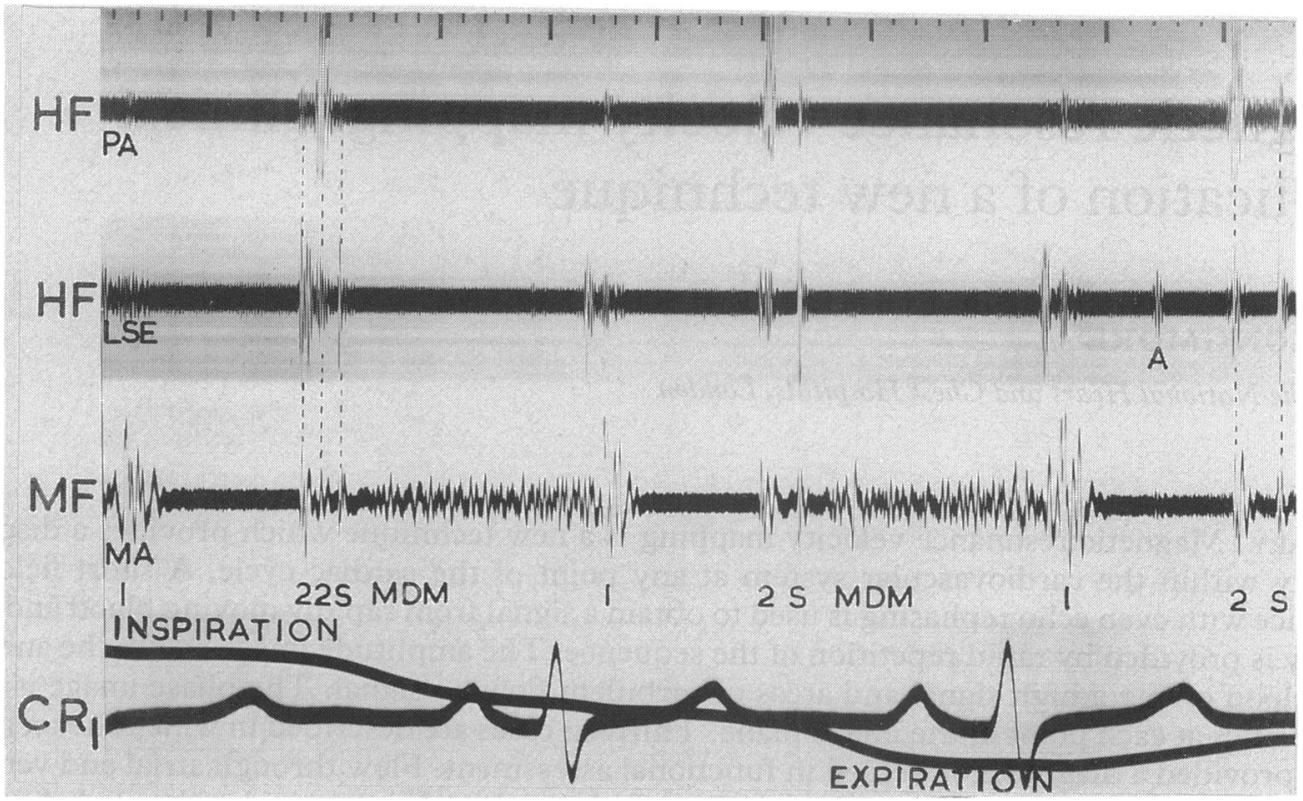

Fig 9 Tracings taken with the National Heart Hospital phonocardiograph showing the value of simultaneous recordings from different areas in a patient with mitral stenosis. In the expiratory phase of respiration the snap (S) might have been $P_{2}$. In inspiration, however, the sequence of $A_{2}$, a loud $P_{2}$ (maximal in the pulmonary area), and a soft opening snap can be easily recognised. High frequency (HF) recordings from the pulmonary area $(P A)$ and lower left sternal edge ( $L S E)$ and a medium frequency recording from the mitral area (MF) to show the mitral diastolic murmur (MDM). Nowadays, identification of sounds would have been achieved, perhaps more scientifically but not more clearly, by echophonocardiography.

the Johns Hopkins Hospital. The sound coincided with the final halt of the upwardly moving valve visualised on the cineaortogram, a point now much more easily confirmed by echocardiography. Thus an aortic ejection sound is produced by movement of an abnormal valve, presumably one with abnormal prominence in systole so that it is carried rapidly upward by ejection, and the sound disappears if the valve is immobilised, particularly by calcium deposition. Aortic root dilatation by itself does not seem to produce a sound and the aortic "root sound" described by Shaver coincides with closure of the tricuspid valve on our high speed echocardiograms. Thus there seems to be no aortic parallel to the pulmonary root sound occurring with pulmonary artery dilatation (though we still do not know the mechanism of the ejection sound with the big aortic root of pulmonary atresia). In aortic regurgitation an ejection sound indicates an abnormal valve (most commonly bicuspid) rather than primary root dilatation which appears to be the commonest cause of aortic regurgitation in the United Kingdom. Rec- ognition of aortic ejection sounds has also been of great value in studying the course of the bicuspid aortic valve and calcific aortic stenosis. A long follow up with Peter Mills of patients with isolated aortic ejection sounds has shown a bicuspid valve as the cause of the sound and in some patients we found that calcification and stenosis developed. We still do not know how often calcification develops and whether it can be prevented.

The evolution of ideas on the origins of heart sounds and murmurs is a striking example of the value of clinical research conducted over a long period of time. In this particular field at least, clinical research has yielded more information than isolated experiments in the laboratory.

I am grateful to Churchill Livingstone for permission to reproduce figs 3, 8, and 9a from Auscultation of the Heart and Phonocardiography by A Leatham, 1975. 